\title{
Polyphyllin VI induces apoptosis and autophagy in human osteosarcoma cells by modulation of ROS/JNK activation
}

This article was published in the following Dove Press journal:

Drug Design, Development and Therapy

\author{
Yong-Liang Yuan ${ }^{1, *}$ \\ Neng Jiang ${ }^{2, *}$ \\ Ze-Yun $\mathrm{Li}^{1}$ \\ Zhi-Zhen Song' \\ Zhi-Heng Yang' \\ Wen-Hua Xue' \\ Xiao-Jian Zhang ${ }^{\prime}$ \\ Yue Du'
}

'Department of Pharmacy, The First Affiliated Hospital of Zhengzhou University, Zhengzhou, Henan, People's Republic of China; ${ }^{2}$ Department of Pharmacy, The Affiliated Tumor Hospital of Guangxi Medical University, Nanning, Guangxi 53002I, People's Republic of China

*These authors contributed equally to this work
Correspondence: Yong-Liang Yuan; Yue Du Department of Pharmacy, The First Affiliated Hospital of Zhengzhou University, Jianshe East Road I,

Zhengzhou, Henan 450052, People's Republic of China

$\mathrm{Tel} / \mathrm{fax}+86037166295651$

Email yylcpu@I26.com;

fccduy@zzu.edu.cn
Purpose: Polyphyllin VI, a main active saponin isolated from traditional medicinal plant Paris polyphylla, has exhibited antitumor activities in several cancer cell lines. In the present study, we investigated the antitumor effect of Polyphyllin VI against human osteosarcoma cells (U2OS) and the underlying molecular mechanisms.

Methods: The U2OS cell lines were used to determine the antiproliferative effect of Polyphyllin VI by CCK8 assay. Cell cycle was analyzed by flow cytometry. The Polyphyllin VI-induced apoptosis was determined by Annexin V-APC/7-AAD apoptosis detection kit and JC-1 staining. Meanwhile, the autophagy was determined by acridine orange staining. The apoptosis and autophagy-related proteins were monitored by Western blot assay. Subsequently, intracellular hydrogen peroxide $\left(\mathrm{H}_{2} \mathrm{O}_{2}\right)$ and the activation of ROS/ JNK pathway were detected.

Results: Polyphyllin VI could potently inhibit cell proliferation by causing G2/M phase arrest. Polyphyllin VI induced mitochondria-mediated apoptosis with the upregulation of proapoptotic proteins Bax and poly ADP-ribose polymerase, and downregulation of antiapoptotic protein Bcl-2 in U2OS cells. Concomitantly, Polyphyllin VI provoked autophagy with the upregulation of critical Atg proteins and accumulation of LC3B-II. Intracellular $\mathrm{H}_{2} \mathrm{O}_{2}$ production was triggered upon exposure to Polyphyllin VI, which could be blocked by ROS scavenger. Polyphyllin VI dramatically promoted JNK phosphorylation, whereas it decreased the levels of phospho-p38 and ERK.

Conclusion: Our results reveal that Polyphyllin VI may effectively induce apoptosis and autophagy to suppress cell growth via ROS/JNK activation in U2OS cells, suggesting that Polyphyllin VI is a potential drug candidate for the treatment of osteosarcomas.

Keywords: Polyphyllin VI, osteosarcoma, apoptosis, autophagy, JNK activation

\section{Introduction}

Osteosarcoma is regarded as the most common primary bone tumor, which stems from primitive bone-forming mesenchymal cells that produce osteoid and/or immature bone. ${ }^{1,2}$ The annual osteosarcoma incidence was at around 23 patients per million and that of adolescence was high at approximately 8-11 per million at 15-19 years of age. ${ }^{3}$ Although current therapies incorporate surgical resection and combinational chemotherapy, the 5-year survival rate for patients with metastatic or relapsed osteosarcoma has remained unchanged over the past 30 years. ${ }^{4}$ Therefore, new treatment approaches for osteosarcoma are urgently needed to be developed. 
Cell death is a trait of cancer that can be classified by morphological differences. Programmed cell death, including apoptosis, autophagy and programmed necrosis, is proposed to be the death of a cell in any pathological format, which is a new target for anticancer therapeutic strategies. ${ }^{5}$ Apoptosis, type I programmed cell death, contains several well-characterized morphological and biochemical changes of dying cells, such as cell volume loss, chromatic condensation, dynamic membrane blebbing and loss of adhesion to the extracellular matrix. ${ }^{6}$ Many studies have reported that apoptosis can be induced by diverse proapoptosis stimuli converging on mitochondria, leading tomitochondrial dysfunction, the activation of caspase enzymes and eventually cell apoptosis. ${ }^{7}$ In particular, it is now well established that numerous chemotherapeutic agents induce tumor cell apoptosis via mitochondria-dependent pathway, ${ }^{8}$ which is highly regulated by Bcl-2 family members. ${ }^{9}$ The Bcl-2 family of proteins is regulated to be the key factors regulating mitochondrial membrane permeabilization. The Bcl-2 family is composed of proapoptotic and antiapoptotic members, such as Bax and Bcl-2. ${ }^{9}$ Autophagy, type II programmed cell death, is a dynamic degradation procedure involving cytoplasmatic vesicles and intracellular organelles are transported into vacuoles called autophagosomes. ${ }^{10,11}$ These organelles or/and vesicles are fused by lysosomes to generate autophagolysosomes, where the mixed material is degraded, leading to cell death. ${ }^{12}$ More importantly, a recent study has highlighted the persistent autophagy served as a potent death signal, which is triggered off in response to the knockout growth factors or on exposure to antitumor agents. ${ }^{13}$ Apoptosis and autophagy are considered as potent therapeutic targets for the treatment of tumor. ${ }^{14}$

Recently, more and more traditional Chinese medicine (TCM) has been applied in osteosarcoma prevention and treatment because of the advantages of curative effect and lower toxicity. ${ }^{15,16}$ Polyphyllin VI, isolated from Paris polyphylla, showed strong anticancer activity among several cancer cell lines. ${ }^{17-19}$ However, the role of Polyphyllin VI in human osteosarcoma cells and the potential mechanisms underlying its antitumor activity remain largely unclear. In this work, we aimed to investigate the molecular mechanism of Polyphyllin VI-induced cell death of human osteosarcoma cells. Exposure of osteosarcoma cells to Polyphyllin VI induced cell apoptosis and autophagy. Moreover, we found the involvement of $\mathrm{H}_{2} \mathrm{O}_{2}$ formation and ROS/JNK activation in the autophagic pathway. Hence, our results revealed the multifunctionality of Polyphyllin VI in its anticancer effect via autophagy and apoptosis in osteosarcoma cells, and it could be a promising drug candidate.

\section{Materials and methods \\ Materials and reagents}

Purified Polyphyllin VI (>98\%) was purchased from Chengdu Must Bio-Technology Co., Ltd (Chengdu, China). Stock solution was dissolved in DMSO (Sigma, St. Louis, MO, USA) and was stored in the dark at $-20^{\circ} \mathrm{C}$. Then, the diluting stock solution was diluted into working concentrations by culture medium immediately before use. CCK 8 (Cell Counting Kit-8) was purchased from Dojindo Molecular Technologies, Inc (Japan). N-acetyl-L-cysteine (NAC) was purchased from Sigma Chemical Co. (St. Louis, MO, USA). Antibodies against poly ADP-ribose polymerase (PARP), Bax, Bcl-2, LC3B-I, LC3B-II, p62, Atg-7, Atg-5, Atg-12-Atg-5, Atg-12, Atg-3, JNK, phosphoJNK, p38, phospho-p38, p-ERK, ERK, caspase-3, caspase-7, and GAPDH were purchased from Cell Signaling Technology (Beverly, MA, USA).

\section{Cell lines and cell culture}

The human osteosarcoma cell lines U2OS were purchased from the Cell Bank of Shanghai Institute of Biochemistry and Cell Biology, Chinese Academy of Sciences (Shanghai, People's Republic of China). The cells were cultured in minimum essential medium (MEM, Gibco Life Technologies) supplemented with 10\% FBS (Sijiqing, Hangzhou, China), $100 \mu \mathrm{g} / \mathrm{mL}$ streptomycin and $100 \mathrm{U} / \mathrm{mL}$ penicillin at $37^{\circ} \mathrm{C}$ with $5 \% \mathrm{CO}_{2}$.

\section{CCK8 assay for cell viability}

The effects of Polyphyllin VI on U2OS cells were analyzed by CCK 8 assay. The U2OS cells were seeded into 96-well plate at the density of $5 \times 10^{3}$ cells/well. After incubation overnight, the cells were treated with Polyphyllin VI $(0,1,2,4,6,8,12$ and $30 \mu \mathrm{M})$ for 24 hrs and 48 hrs. Following the treatment, cells were incubated with $0.5 \%$ CCK 8 for 4 hrs. The formed formazan crystals were dissolved in DMSO $(200 \mu \mathrm{L} /$ well $)$. The absorbance was measured at a test wavelength of $570 \mathrm{~nm}$ and a reference wavelength of $650 \mathrm{~nm}$.

\section{APC annexin V/7-AAD staining assay for cell apoptosis}

Apoptotic cells were evaluated by low cytometry. U2OS cells were treated with various concentrations of Polyphyllin VI or 
$0.1 \%$ DMSO for $24 \mathrm{hrs}$. For flow cytometry, $1 \times 10^{6} \mathrm{U} 2 \mathrm{OS}$ cells in $500 \mu \mathrm{L}$ binding buffer were stained with $5 \mu \mathrm{L}$ Annexin $\mathrm{V}-\mathrm{APC}$ and $5 \mu \mathrm{L}$ 7-AAD at room temperature for $15 \mathrm{mins}$, respectively. Cells were then analyzed by flow cytometry using BD FACSCalibur flow cytometer (Becton \& Dickinson Company, Franklin Lakes, NJ, USA).

\section{Mitochondrial membrane potential measurement}

The mitochondrial membrane potential (MMP) was determined using a JC-1 assay kit (Nanjing KeyGen Biotech Co., Ltd., Nanjing, China). Cells were treated with Polyphyllin VI $(0,2.5,5$, and $7.5 \mu \mathrm{M})$ for $24 \mathrm{hrs}$. After collection, the U2OS cells were washed with PBS twice, stained with $500 \mathrm{~mL} \mathrm{JC}-1$ in a $37^{\circ} \mathrm{C}, 5 \% \mathrm{CO}_{2}$ incubator for 25 mins, protected from light. And the cells were then centrifuged at $2000 \mathrm{rpm} \times 5 \mathrm{mins}$ and washed twice. The cells were resuspended in $500 \mathrm{~mL} 1 \times$ incubation buffer for flow cytometry analysis $(488 \mathrm{~nm}$ excitation and $525 \mathrm{~nm}$ emission filters).

\section{Acridine orange staining for cell autophagy}

For the detection of autophagy, U2OS cells were treated with Polyphyllin VI or $0.1 \%$ DMSO for 24 hrs. Following treatment, the cells were stained with $1 \mu \mathrm{g} / \mathrm{mL}$ acridine orange (Amresco, Solon, OH, USA) in PBS for 30 mins at $37^{\circ} \mathrm{C}$. Cells were then observed using the BD Accuri $\mathrm{C} 6$ flow cytometry.

\section{Hydrogen peroxide $\left(\mathrm{H}_{2} \mathrm{O}_{2}\right)$ measurement}

For quantitative detection of intracellular $\mathrm{H}_{2} \mathrm{O}_{2}$ levels, U2OS cells were pretreated with treated with Polyphyllin $\mathrm{VI}(0,2.5,5$, and $7.5 \mu \mathrm{M})$ for $24 \mathrm{hrs}$ and then incubated with 2',7'-dichlorofluorescin-diacetate (DCHF-DA) (Invitrogen, Carlsbad, CA, USA) according to the manufacturer's protocols. After incubation with $10 \mu \mathrm{M}$ DCHF-DA for 30 mins at $37^{\circ} \mathrm{C}$ in dark, DCFH-DA was cleaved by intracellular esterase to liberate free DCFH. The relative $\mathrm{H}_{2} \mathrm{O}_{2}$ levels of the cells were measured by flow cytometry.

\section{Western blotting analysis}

After U2OS cells were incubated with Polyphyllin VI (0, $2.5,5$, and $7.5 \mu \mathrm{M}$ ) for $24 \mathrm{hrs}$, the total proteins were extracted and quantified using a BCA Protein Assay Kit (Beyotime, Shanghai, China). The proteins were separated on SDS-PAGE gels with $10 \%$ Tris-SDS gel, and wet- transferred to NC membrane (BioRad Laboratories, Hercules, CA, USA) and blotted with primary antibodies specific for PARP, Bax, Bcl-2, LC3B-I, LC3B-II, p62, Atg-7, Atg-5, Atg-12-Atg-5, Atg-12, Atg-3, JNK, phospho-JNK, p38, phospho-p38, ERK, p-ERK, caspase-3, caspase-7, GAPDH, etc. The proteins were probed with using anti-rabbit $(1: 5,000)$ or anti-mouse $(1: 5,000)$ secondary antibodies tagged with horseradish peroxidase. Bound immunocomplexes were detected using the ChemiDoc XRS (BioRad).

\section{Statistical analysis}

All experiments were performed at least three times. The results were analyzed using one-way ANOVA with Tukey multiple comparison. All data were expressed as the mean \pm SD. $P<0.05$ was regarded as a statistically significant difference.

\section{Results}

\section{Polyphyllin VI inhibits the proliferation and induces cell cycle G2/M arrest in OS cells}

Figure 1A shows the chemical structure and molecular weight of the Polyphyllin VI. To investigate the inhibitory effects of Polyphyllin VI on growth, U2OS cells were exposed to various concentrations of Polyphyllin VI for $24 \mathrm{hrs}$ and $48 \mathrm{hrs}$ by CCK 8 assay.

The $\mathrm{IC}_{50}$ values of Polyphyllin VI were $7.33 \pm 2.85 \mu \mathrm{M}$ for $24 \mathrm{hrs}$ and $6.02 \pm 1.42 \mu \mathrm{M}$ for $48 \mathrm{hrs}$ (Figure 1B). The results showed that Polyphyllin VI had a significant proliferation inhibitory effect on osteosarcoma cell lines in a dose- and time-dependent manner. In addition, in order to investigate the causal relationship between cell proliferation inhibition and cell cycle arrest by Polyphyllin VI, we performed the cell cycle distribution detection. As shown in Figure S1, G2/M phase cell number after 24-hr treatment by Polyphyllin VI was increased; on the contrary, the number of cells in $\mathrm{S}$ and $\mathrm{G} 0 / \mathrm{G} 1$ phase was decreased. The above results indicated that Polyphyllin VI inhibited cell proliferation by trigging the G2/M-phase arrest.

\section{Polyphyllin VI induces mitochondria- mediated apoptosis}

To determine whether apoptosis is responsible for the cell growth inhibition, we used an Annexin V-APC/7-AAD doubled-labeled flow cytometry analysis to ascertain whether Polyphyllin VI treatment elicited apoptosis in 


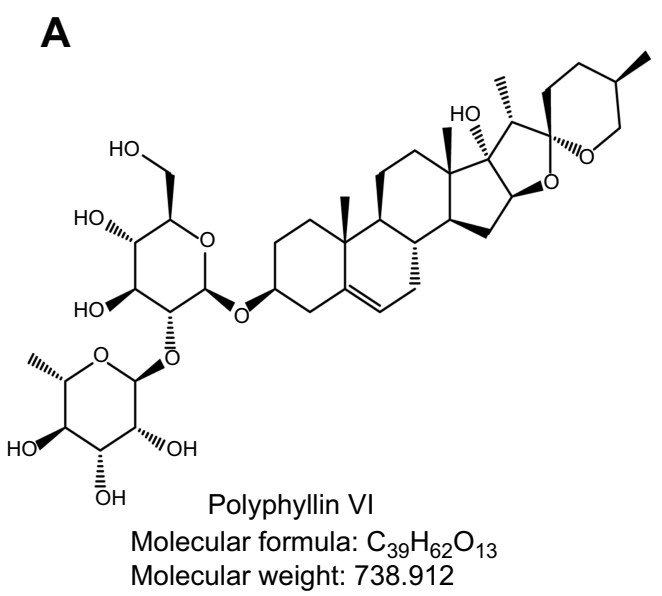

B

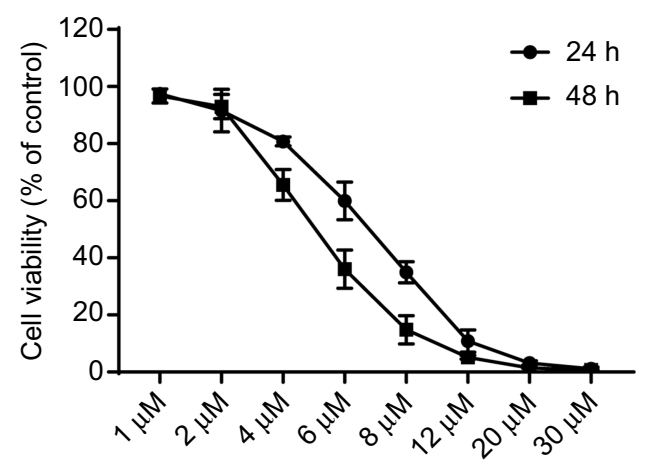

Polyphyllin VI concentration $(\mu \mathrm{M})$

Figure I (A) the structure and molecular weight of Polyphyllin VI. (B) U2OS cells were treated with Polyphyllin VI, and then the cell viability was determined by the CCK8 assay at the indicated time.

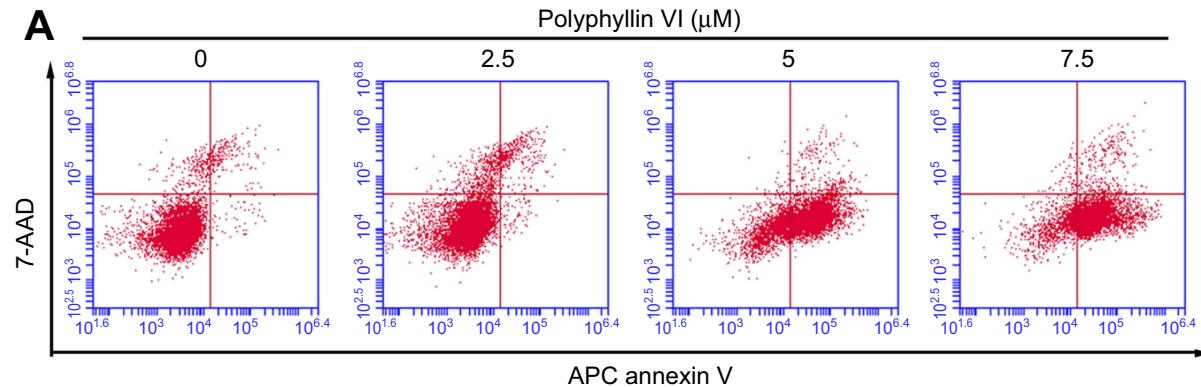

B

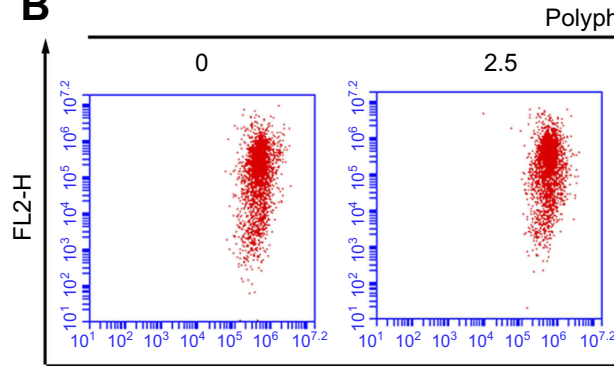

Polyphyllin VI $(\mu \mathrm{M})$

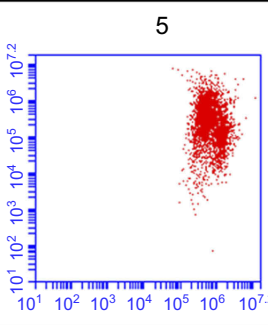

FL1-H

10.7

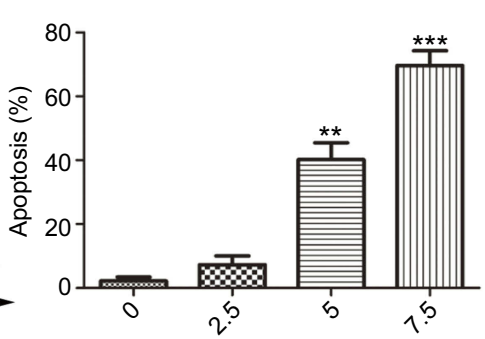

Polyphyllin VI concentration $(\mu \mathrm{M})$
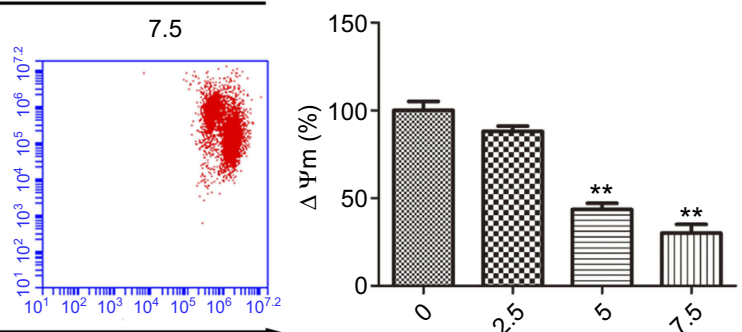

Polyphyllin VI concentration $(\mu \mathrm{M})$

C
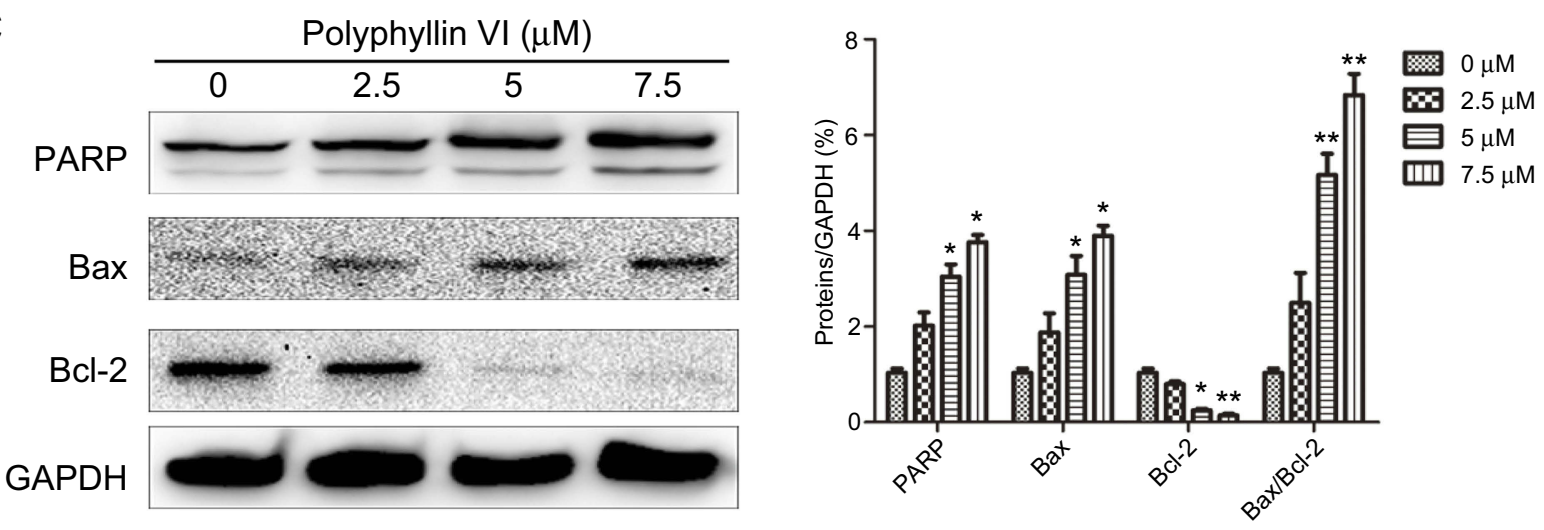

Figure 2 Polyphyllin VI induces apoptosis in osteosarcomas cells. (A) U2OS cells were incubated with the indicated concentrations of Polyphyllin VI for 24 h and stained with Annexin V-APC and 7-AAD. (B) Flow cytometric measurement of $\Delta \psi \mathrm{m}$ in U2OS cells after treatment with Polyphyllin VI (2.5, $5,7.5 \mu \mathrm{M})$ for $24 \mathrm{~h}$. (C) Western blot analysis with antibodies specific for cleaved PARP, Bcl-2 and Bax was used as the loading control. Results are presented as the mean \pm SD from three independent experiments. $* \mathrm{P}<0.05$, $* * \mathrm{P}<0.0 \mathrm{I}$, compared to control. 
osteosarcoma cells. The results showed that the proportion of apoptosis was negligible for control cells, whereas the apoptotic rate of osteosarcomas cells increased in a dosedependent manner after being treated with Polyphyllin VI for 24 hrs (Figure 2A).

Since loss of MMP was associated with the initiation and activation of the apoptotic process in cells, we investigated the effect of Polyphyllin VI on the integrity of the mitochondrial membrane (Figure $2 \mathrm{~B}$ ). The results revealed that MMP significantly decreased following Polyphyllin VI treatment, suggesting that Polyphyllin VI induces the apoptosis in osteosarcoma cells through disruption of their mitochondrial dysfunction.

We then investigated the levels of proteins related to apoptosis by Western blot. As shown in Figure 2C, the expression of antiapoptotic protein Bcl-2 in U2OS dramatically decreased in a dose-dependent manner, whereas the protein levels of proapoptotic proteins Bax and cleaved PARP gradually increased. Besides, Figure S2 showed an obvious decrease in the activation of Caspase- 3 and Caspase-7 in U2OS cells. These results suggested that Polyphyllin VI induces apoptotic cell death in U2OS cells via the mitochondrial mediated apoptotic pathway.

\section{Polyphyllin VI triggers autophagy in osteosarcomas cells}

Autophagy is a form of programmed cell survival, which affects tumor initiation and progression. ${ }^{20}$ To further understand whether autophagy is involved in the Polyphyllin VIinduced cell growth inhibition, we measured the autophagic response to Polyphyllin VI in osteosarcomas cells. After exposure to Polyphyllin VI, the cells were stained with acridine orange and analyzed by flow cytometry (Figure 3A). The results showed that fluorescence intensity was significantly increased in U2OS cells after 24-hr exposure to Polyphyllin VI, which indicated Polyphyllin VI triggered autophagy in osteosarcomas cells. During autophagy, microtubule-associated protein 1 light chain 3 (LC3) is cleaved to LC3-I, which is further modified to another form, called LC3-II (Figure 3B). LC3-II is localized to the autophagosomal membrane, which is now identified as a marker for autophagosome and autophagy activity. P62 is an autophagy substrate that is also used as a marker of autophagy activity. The increased expression of IC3-II and p62 was observed in a dose-dependent manner up to $7.5 \mu \mathrm{M}$ Polyphyllin VI (Figure 3B). These data were consistent with the results of other autophagy marker proteins, including Atg-5, Atg-7 and Atg-3 (Figure 3C).
Polyphyllin VI increased the expression of Atg-5 and Atg-7 in a dose-dependent manner, whereas the increasing trend of the expression of Atg-3 was terminated when cells were treated with $7.5 \mu \mathrm{M}$ Polyphyllin VI. Interestingly, the expression of Atg-5 was increased while that of Atg-12 was decreased, which indicated an increased expression of Atg-5-Atg-12 conjugation in osteosarcomas cells after 24-hr exposure to Polyphyllin VI. In brief, our data suggest that Polyphyllin VI caused autophagy in osteosarcoma cells.

\section{Intracellular $\mathrm{H}_{2} \mathrm{O}_{2}$ and JNK activation involve in polyphyllin VI-induced autophagy and apoptosis}

We further investigated the molecular mechanisms underlying the ability of Polyphyllin VI to induce autophagy and apoptosis in osteosarcomas cells. Both autophagy and apoptosis can be triggered by common upstream signals, such as excessive intracellular $\mathrm{H}_{2} \mathrm{O}_{2}$, which was involved in the anticancer activity of many chemotherapy drugs. Thus, we examined the $\mathrm{H}_{2} \mathrm{O}_{2}$ activation of osteosarcomas cells after exposure to Polyphyllin VI. As expected, Polyphyllin VI resulted in a significant increase in intracellular $\mathrm{H}_{2} \mathrm{O}_{2}$ production, as shown by FACS detection assay (Figure 4A). Preincubation of cells with the ROS scavenger NAC completely blocked the increase in intracellular $\mathrm{H}_{2} \mathrm{O}_{2}$ production (Figure 4B), suggesting ROS species controlled by Polyphyllin VI-induced autophagy and apoptosis.

ROS has been shown as a strong signal for the regulator of mitogen-activated protein kinases (MAPK) family members including ERK, p38 and JNK, which may subsequently mediate antioxidant responses to induce autophagy and apoptosis (Figure 4C). To determine the role of these kinases in Polyphyllin VI-induced autophagy and apoptosis, we evaluated the levels of these proteins by Western blot. The results showed that levels of JNK phosphorylation was dramatically increased in Polyphyllin VItreated osteosarcomas cells in a dose-dependent manner, whereas the levels of phospho-p38 and ERK decreased when cells were treated with $7.5 \mu \mathrm{M}$ Polyphyllin VI. In brief, these results showed that JNK activation involved in Polyphyllin VI-induced autophagy and apoptosis in osteosarcomas cells.

\section{Discussion}

Polyphyllin VI is a main active saponin isolated from traditional medicinal plant Paris polyphyllin, which 
A
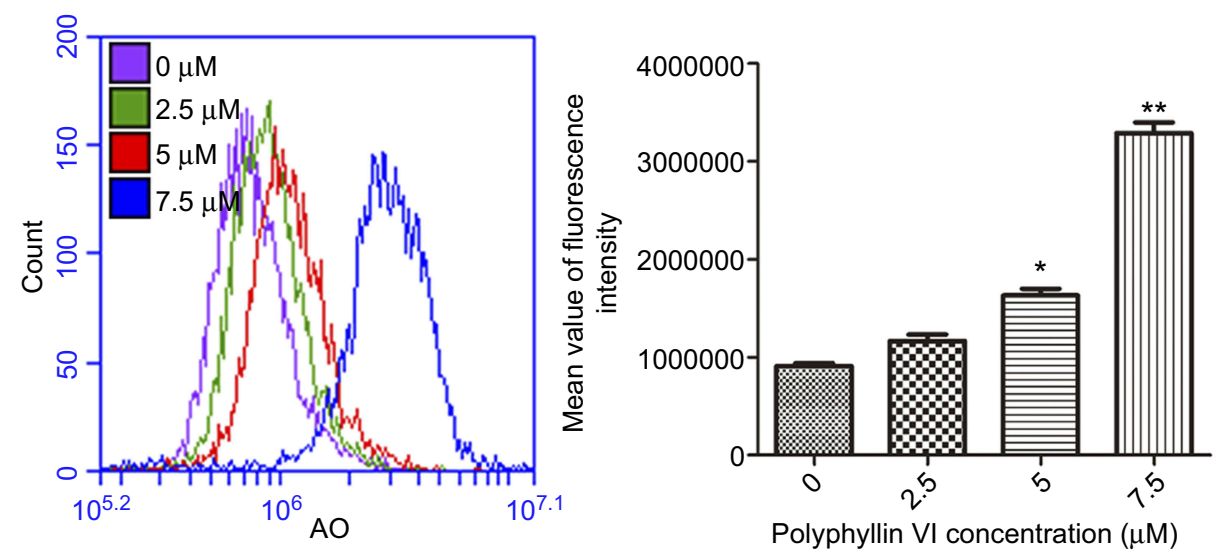

B
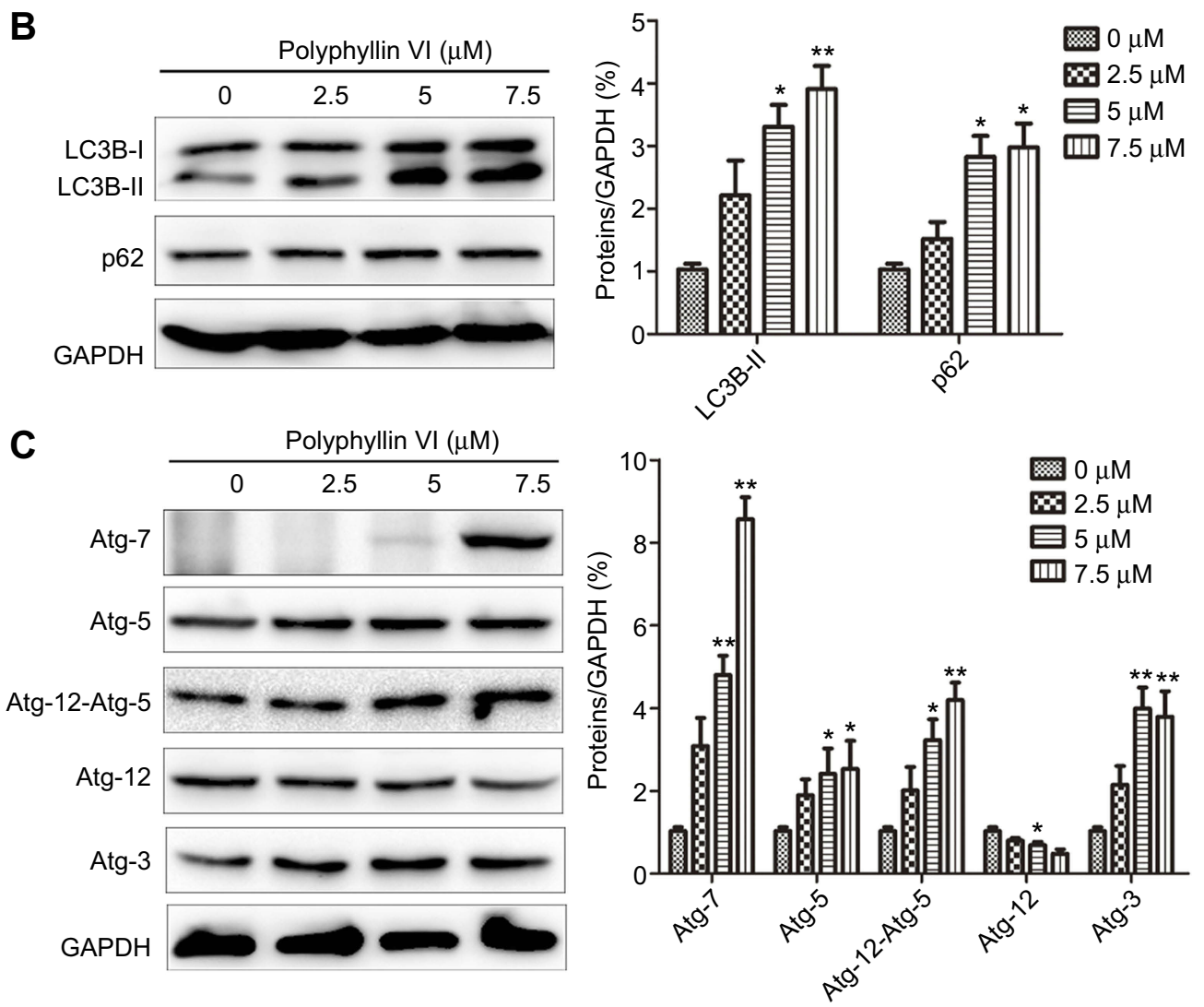

Figure 3 Polyphyllin VI triggers autophagy in osteosarcomas cells. (A) U2OS cells were incubated with 0-7.5 $\mu$ M Polyphyllin VI to detect the formation of AVOs performed by AO staining. (B) Western blot displays expression levels of LC3-I, LC3-II and p62 in Polyphyllin VI-treated U2OS cells. (C) Western blot displays expression levels of Atg3, Atg-5, Atg-12-Atg-5 complex, Atg-12 free and Atg-7 in Polyphyllin VI-treated U2OS cells. Results are presented as the mean \pm SD from three independent experiments. $* \mathrm{P}<0.05, * * \mathrm{P}<0.01$, compared to control.

shows antitumor activity against lung cancer A549 and NCI-H1299 cell lines, ${ }^{17}$ breast cancer cell MCF-7 and MDA-MB-231 cell lines, ${ }^{18}$ and hepatocellular carcinoma HepG2 cell line. ${ }^{19}$ The present study for the first time investigated the cell growth inhibition of Polyphyllin VI on human osteosarcoma cancer cells.

As shown in Figure 5, it is well displayed that the mechanism of Polyphylliin VI-induced apoptosis and autophagy in U2OS cells. Polyphyllin VI-induced cell death was associated with both apoptosis (loss of MMP, decreased levels of antiapoptotic protein Bcl-2 and increased levels of proapoptotic proteins Bax and cleaved PARP) and autophagy (the notable induction of autophagic markers such as conversion of LC3-I to LC3-II, accumulation of Atg-5, Atg-7 and incorporation of Atg-12-Atg-5 protein complex).

Apoptosis is one of the most important forms of cell death and crucially involved in tumor formation and treatment response. Most conventional chemotherapies and 

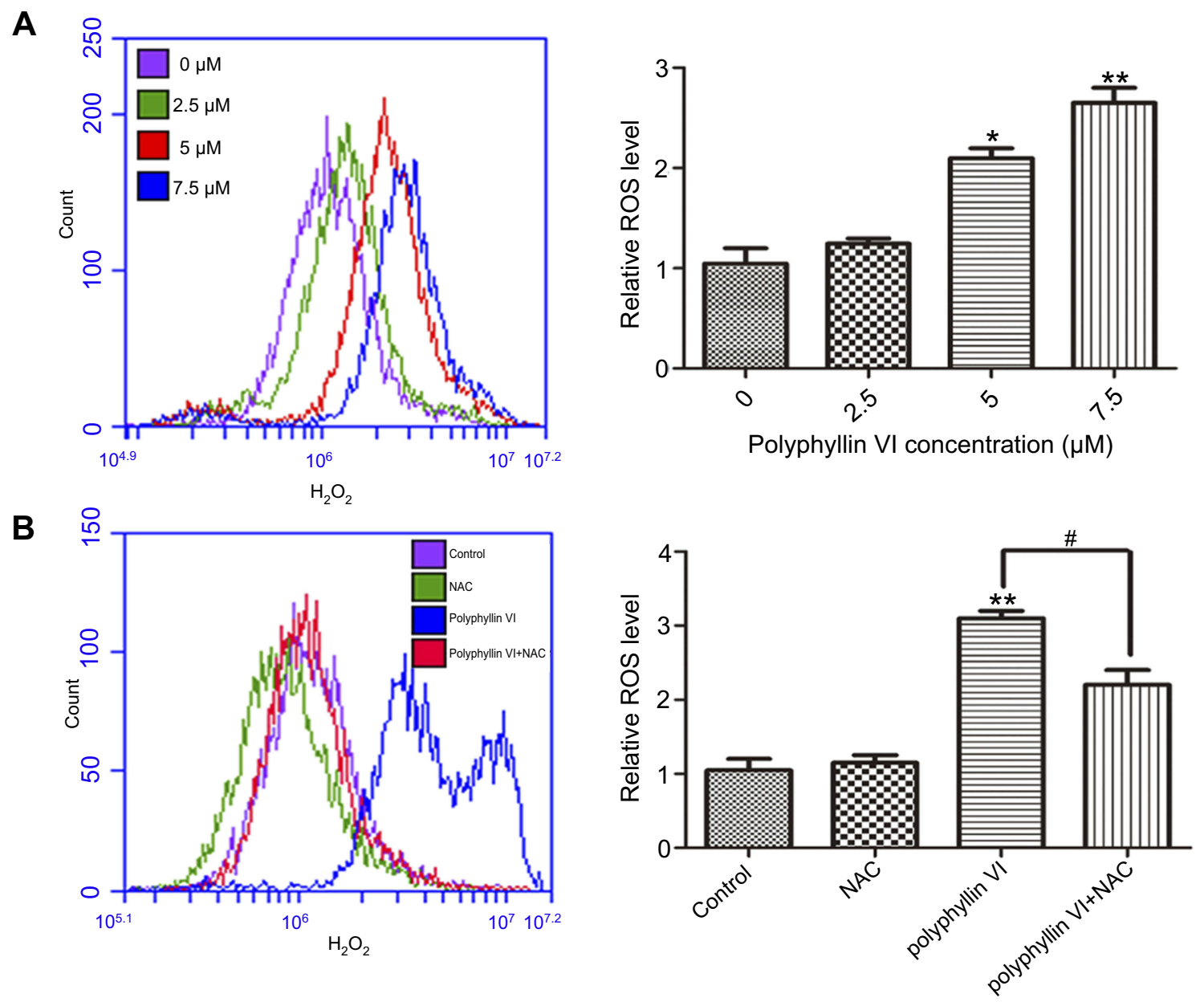

C
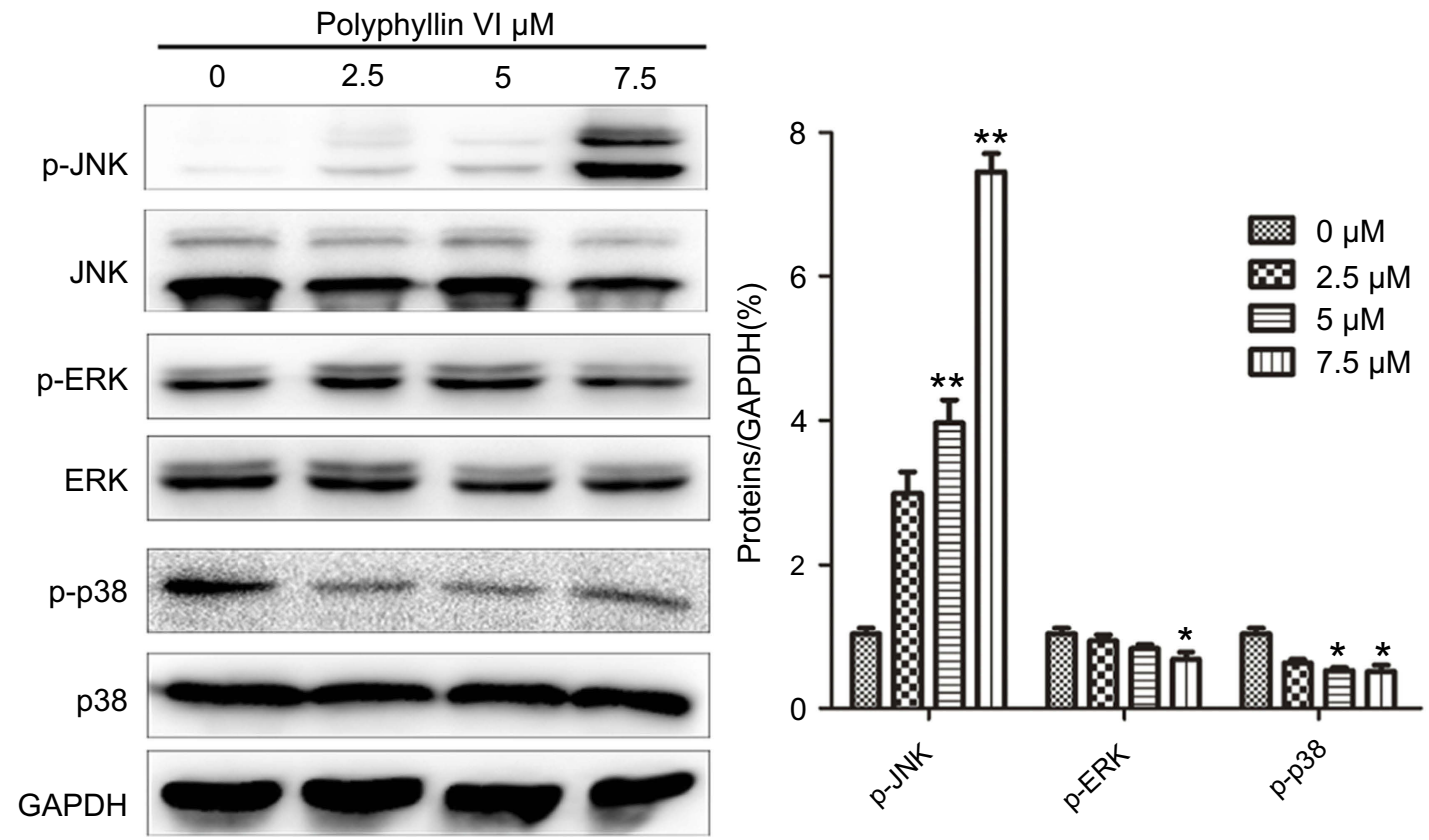

Figure 4 Intracellular $\mathrm{H} 2 \mathrm{O} 2$ and JNK activation involved in Polyphyllin VI-induced autophagy and apoptosis. (A) U2OS cells were incubated with the indicated concentrations of Polyphyllin VI for $24 \mathrm{~h}$. The $\mathrm{H} 2 \mathrm{O} 2$ level was measured by flow cytometry analysis. (B) Cells were treated with $7.5 \mu \mathrm{M}$ Polyphylliin VI alone or in combination with the ROS scavenger NAC ( $10 \mathrm{mM})$, and then cells were subjected to flow cytometry analysis of intracellular $\mathrm{H} 2 \mathrm{O} 2$. (C) Western blot analysis of p-JNK, JNK, p-ERK, ERK, P-p38 and p38 in cells treated with Polyphyllin VI at the indicated doses for $24 \mathrm{~h}$. *P<0.05, **P<0.0I, compared to control. 


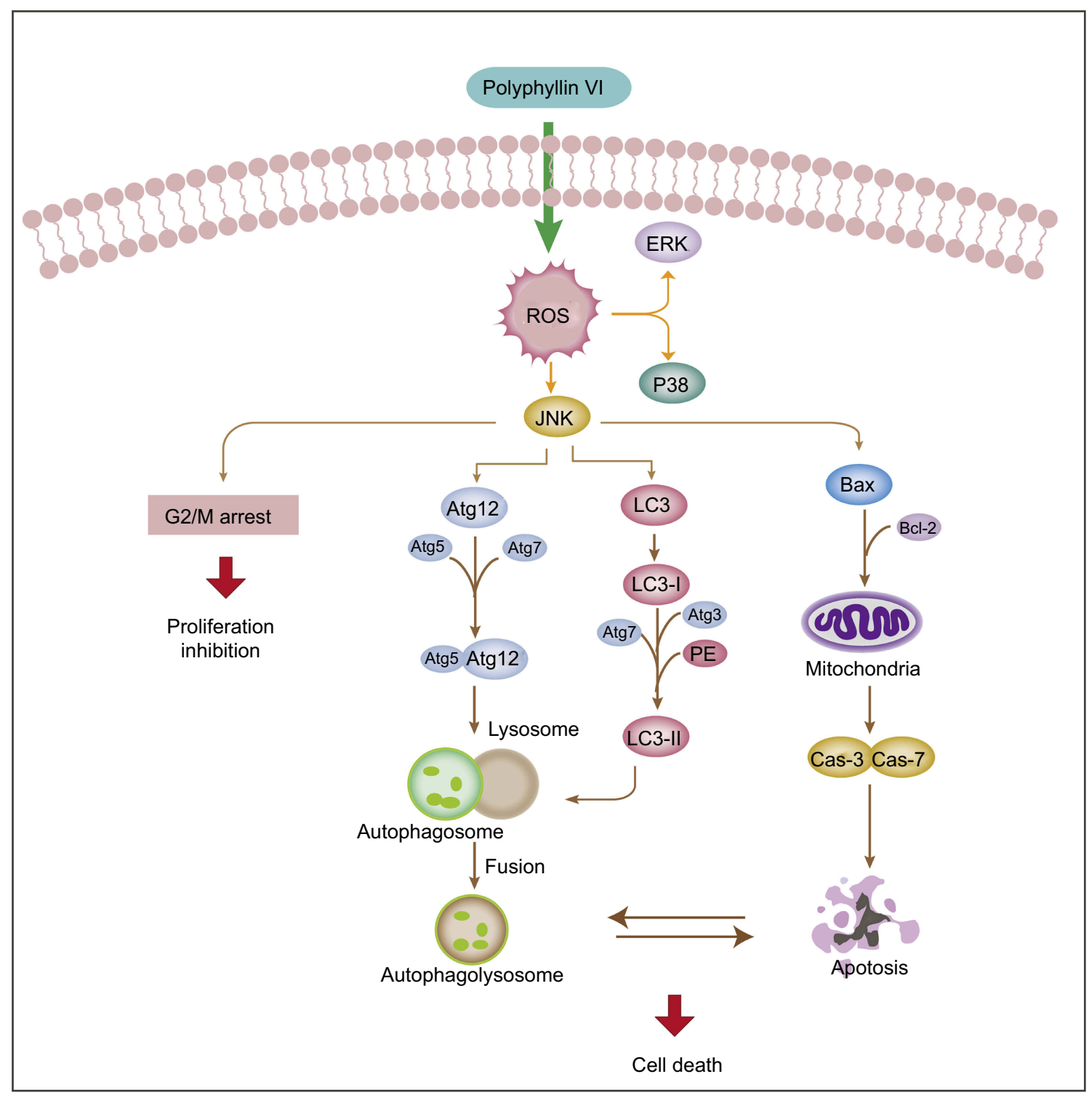

Figure 5 The mechanism of Polyphyllin VI-induced apoptosis and autophagy in U2OS cells.

radiotherapy rely on activation of apoptosis pathways to provoke apoptotic cell death in cancer cells. ${ }^{21}$ Poly PARP is a nuclear DNA-binding protein, which plays an important in DNA repair. ${ }^{22}$ Its cleavage by caspase- 3 is inactivated and is a hallmark of apoptosis. ${ }^{23}$ Our results showed that Polyphyllin VI induced apoptosis by observation of PARP cleavage in osteosarcomas cells. This finding is in agreement with flow cytometry analysis demonstrating that the number of apoptotic cells is increased in Polyphyllin VI-treated cells. Analysis of other proteins in apoptosis pathways, such as Bcl-2 and Bax, also confirmed the occurrence of apoptosis. Downregulation of Bcl-2 is companied by overexpression of Bax in osteosarcoma cell with treatment of Polyphyllin VI. As proapoptotic Bax activation can lead to mitochondrial membrane permeabilization, our results suggest that Polyphyllin VI initiates apoptotic cascade through the mitochondrial pathway. The results are consistent with finding that Polyphyllin VI disrupted the integrity of the mitochondrial membrane in osteosarcomas cells. These data indicated that Polyphyllin VI regulated apoptosis by the balance of pro- and antiapoptotic proteins in human osteosarcomas U2OS cell line.

Autophagy is an essential process by which cellular materials are degraded to provide energy and macromolecular precursors, as well as avoid cytotoxic product accumulation. $^{24,25}$ Thus, autophagy critically regulates cellular homeostasis with a balance between cell survival and cell death. In tumor cells, some chemotherapies and 
radiotherapies induce autophagy, ${ }^{26}$ which results in the emission of danger signals and the initiation of a therapeutically relevant anticancer immune response. ${ }^{14}$ Autophagy involves a sequential recruitment of many proteins that orchestrate the different steps of autophagy. The ability of Polyphyllin VI to trigger autophagy was confirmed by the enhanced conversion of LC3B-I to LC3B-II and increased expression of p62, Atg-3, Atg-5 and Atg-7. In addition, Polyphyllin VI decreased the expression of $\operatorname{Atg} 12$ and increased expression of Atg12-Atg5, which is an important ubiquitin-like conjugate. ${ }^{27}$ Thus, our results showed that Polyphyllin VI-induced autophagy might be responsible for the inhibition of cell growth in human osteosarcomas U2OS cell line.

A lot of studies have demonstrated a very complex association between apoptosis and autophagy, which may promote each other, inhibit each other, or unilaterally promote or inhibit. ${ }^{28}$ Apoptosis-related caspase family proteins interact with autophagy-related proteins, and some studies have shown that caspase- 3 inhibits autophagy to promote apoptosis by cleavage of Beclin- $1 .{ }^{29,30}$ Bcl-2 family proteins play a key dual regulatory role in apoptosis and autophagy. ${ }^{29}$ Studies have shown that the antiapoptotic proteins $\mathrm{Bcl}-2$ and $\mathrm{Bcl}-\mathrm{xL}$ do not directly affect autophagy, but indirectly through inhibition of Bax and Bak. ${ }^{31}$ Autophagosome formation is dependent on the covalent attachment of a series of Atg proteins during protein ubiquitination. Atg5 and Atg12 are known as the "core" of autophagy and are required for autophagosome formation. They have also been found to be involved in the regulation of apoptosis. Atg5 can be cleaved by Caplains, causing the Atg5 N-terminal fragment to translocate to the mitochondria in an unknown mechanism, binding to the antiapoptotic protein $\mathrm{Bcl}-\mathrm{xL}$, promoting mitochondrial cytochrome $\mathrm{C}$ release and inducing apoptosis. $^{32}$ Atg12 was developed to have a proapoptotic function due to its $\mathrm{BH} 3$-like domain that binds to $\mathrm{Bcl}-2$ and Mcl-1. ${ }^{33}$ According to the relevant references ${ }^{34-36}$, the upregulated expression of LC3B-II and Atg5-Atg12 showed that cell death caused by autophagic pathway when apoptosis was blocked. In contrast, when suppression of autophagy diminished, the increased expression of Bax and decreased expression of Bcl-2 indicated that apoptosis is promoted to lead to cell death. Further experimental research was needed to study about the molecular mechanism between apoptosis and autophagy.

ROS has been described as the chemical species that are produced by incomplete redox reaction and includes the superoxide anion $\left(\mathrm{O}_{2}-\right)$, hydrogen peroxide $\left(\mathrm{H}_{2} \mathrm{O}_{2}\right)$ and the hydroxyl radical $(\mathrm{HO} \bullet){ }^{37}$ In this study, we found that Polyphyllin VI increased intracellular $\mathrm{H}_{2} \mathrm{O}_{2}$ production, which could be blocked by ROS scavenger NAC. In apoptosis-inducing processes, ROS production is due to the electron transport chain in mitochondria and to the MMP decrease, leading to the mitochondrial translocation of Bax, Bcl-2 and Cytochrome C. ${ }^{38,39}$ Besides, the autophagy-inducing activity processes are proved to be related to the accumulation of intracellular ROS, which have been functioning as signaling molecules in the autophagic pathway through reversibly oxidizing crucial signaling components. ${ }^{40}$ Thus, our results suggested that $\mathrm{H}_{2} \mathrm{O}_{2}$ was involved in Polyphyllin VI-induced autophagy and apoptosis.

ROS has been shown as an inducer or mediator for the activation of MAPK family members, ${ }^{41}$ which play an important role in autophagy and apoptosis. Exposure of osteosarcomas cells to Polyphyllin VI led to strong activation of JNK (phosphorylation), suggesting that Polyphyllin VIinduced cell growth inhibition might be JNK independent, as phospho-p38 and ERK had no significant enhancement. JNK activation has been implicated in apoptosis and autophagy in response to a variety of stress signals. ${ }^{42} \mathrm{JNK}$ could mediate antiapoptotic proteins $\mathrm{Bcl}-2 / \mathrm{Bcl}-\mathrm{xL}$ phosphorylation to change the $\mathrm{MMP}^{43}$; in this regard, Polyphyllin VI-induced disruption of mitochondrial dysfunction might be associated with JNK-mediated apoptotic response. JNK activation also contributed to autophagic induction, which is a key part for upregulation of LC3. Consistently, we also found that Polyphyllin VI-induced activation of JNK pathway was accompanied by upregulation of LC3B-II in human osteosarcomas U2OS cell line. ROS is the upstream pathway of JNK and is probably the core of the effect of Polyphyllin VI, which mediates G2/M arrest, apoptosis and autophagy by activating ROS/JNK pathway.

In summary, our study demonstrated that Polyphyllin VI could inhibit cell growth of human osteosarcomas cancer cell line by induction of autophagy and apoptosis, which was associated with accumulation of intracellular $\mathrm{H}_{2} \mathrm{O}_{2}$ and activation of JNK pathway. This study revealed antitumor effects of Polyphyllin VI and its potential for the treatment of osteosarcomas.

\section{Acknowledgments}

The authors gratefully acknowledge the financial supports by the Program of the National Natural Science Foundation of China (grant numbers 81903874, 81603287 and 81703331), as well as the Program of Natural Science Foundation of Guangxi 
Province of China (grant number 2017GXNSFAA198077) and Key Research Projects of Henan Higher Education Institutions(20B360018, 20A350010).

\section{Disclosure}

The authors report no conflicts of interest in this work.

\section{References}

1. Ottaviani G, Jaffe N. The epidemiology of osteosarcoma. Cancer Treat Res. 2009;152:3-13. doi:10.1007/978-1-4419-0284-9_1

2. Li J, Yang Z, Li Y, et al. Cell apoptosis, autophagy and necroptosis in osteosarcoma treatment. Oncotarget. 2016;7(28):44763-44778. doi:10.18632/oncotarget.8206

3. Bielack S, Carrle D, Casali PG. Osteosarcoma: ESMO clinical recommendations for diagnosis, treatment and follow-up. Ann Oncol. 2009;20(Suppl 4):137-139. doi:10.1093/annonc/mdp154

4. Kansara M, Teng MW, Smyth MJ, Thomas DM. Translational biology of osteosarcoma. Nat Rev Cancer. 2014;14(11):722-735. doi:10.1038/ $\operatorname{nrc} 3838$

5. Ouyang L, Shi Z, Zhao S, et al. Programmed cell death pathways in cancer: a review of apoptosis, autophagy and programmed necrosis. Cell Prolif. 2012;45(6):487-498. doi:10.1111/j.1365-2184.2012.00845.x

6. Valentin R, Grabow S, Davids MS. The rise of apoptosis: targeting apoptosis in hematologic malignancies. Blood. 2018;132(12):12481264. doi:10.1182/blood-2018-02-791350

7. Nagata S. Apoptosis and clearance of apoptotic cells. Annu Rev Immunol. 2018;36:489-517. doi:10.1146/annurev-immunol-042617-053010

8. Sinha K, Das J, Pal PB, Sil PC. Oxidative stress: the mitochondriadependent and mitochondria-independent pathways of apoptosis. Arch Toxicol. 2013;87(7):1157-1180. doi:10.1007/s00204-013-1034-4

9. Czabotar PE, Lessene G, Strasser A, Adams JM. Control of apoptosis by the BCL-2 protein family: implications for physiology and therapy. Nat Rev Mol Cell Biol. 2014;15(1):49-63. doi:10.1038/nrm3722

10. Mizushima N, Yoshimori T, Ohsumi Y. The role of Atg proteins in autophagosome formation. Annu Rev Cell Dev Biol. 2011;27:107132. doi:10.1146/annurev-cellbio-092910-154005

11. Mizushima N. Autophagy: process and function. Genes Dev. 2007;21 (22):2861-2873. doi:10.1101/gad.1599207

12. Levy JMM, Towers CG, Thorburn A. Targeting autophagy in cancer. Nat Rev Cancer. 2017;17(9):528-542. doi:10.1038/nrc.2017.53

13. Jutten B, Keulers TG, Schaaf MB, et al. EGFR overexpressing cells and tumors are dependent on autophagy for growth and survival. Radiother Oncol. 2013;108(3):479-483. doi:10.1016/j.radonc.2013.06.033

14. Galluzzi L, Bravo-San Pedro JM, Levine B, Green DR, Kroemer G. Pharmacological modulation of autophagy: therapeutic potential and persisting obstacles. Nat Rev Drug Discov. 2017;16(7):487-511. doi:10.1038/nrd.2017.22

15. Yen JH, Huang ST, Huang HS, et al. HGK-sestrin 2 signalingmediated autophagy contributes to antitumor efficacy of Tanshinone IIA in human osteosarcoma cells. Cell Death Dis. 2018;9(10):1003. doi:10.1038/s41419-018-1016-9

16. Liu Z, Zheng Q, Chen W, et al. Chemosensitizing effect of Paris Saponin I on Camptothecin and 10-hydroxycamptothecin in lung cancer cells via p38 MAPK, ERK, and Akt signaling pathways. Eur J Med Chem. 2017;125:760-769. doi:10.1016/j.ejmech.2016.09.066

17. Lin Z, Liu Y, Li F, et al. Anti-lung cancer effects of polyphyllin VI and VII potentially correlate with apoptosis in vitro and in vivo. Phytother Res. 2015;29(10):1568-1576. doi:10.1002/ptr.5430

18. Lee MS, Yuet-Wa JC, Kong SK, et al. Effects of polyphyllin D, a steroidal saponin in Paris polyphylla, in growth inhibition of human breast cancer cells and in xenograft. Cancer Biol Ther. 2005;4 (11):1248-1254. doi:10.4161/cbt.4.11.2136
19. Ong RC, Lei J, Lee RK, et al. Polyphyllin D induces mitochondrial fragmentation and acts directly on the mitochondria to induce apoptosis in drug-resistant HepG2 cells. Cancer Lett. 2008;261(2):158164. doi:10.1016/j.canlet.2007.11.005

20. Fitzwalter BE, Thorburn A. Recent insights into cell death and autophagy. Febs J. 2015;282(22):4279-4288. doi:10.1111/febs.13515

21. Hassan M, Watari H, AbuAlmaaty A, Ohba Y, Sakuragi N. Apoptosis and molecular targeting therapy in cancer. Biomed Res Int. 2014;2014:150845. doi:10.1155/2014/150845

22. Morales J, Li L, Fattah FJ, et al. Review of poly (ADP-ribose) polymerase (PARP) mechanisms of action and rationale for targeting in cancer and other diseases. Crit Rev Eukaryot Gene Expr. 2014;24(1):15-28. doi:10.1615/CritRevEukaryotGeneExpr.2013006875

23. Chaitanya GV, Steven AJ, Babu PP. PARP-1 cleavage fragments: signatures of cell-death proteases in neurodegeneration. Cell Commun Signal. 2010;8:31. doi:10.1186/1478-811X-8-31

24. Jiang X, Overholtzer M, Thompson CB. Autophagy in cellular metabolism and cancer. J Clin Invest. 2015;125(1):47-54. doi:10.1172/JCI73942

25. Wang Y, Qin ZH. Coordination of autophagy with other cellular activities. Acta Pharmacol Sin. 2013;34(5):585-594. doi:10.1038/aps.2012.194

26. Zeng X, Kinsella TJ. Impact of autophagy on chemotherapy and radiotherapy mediated tumor cytotoxicity: "to live or not to live". Front Oncol. 2011;1:30. doi:10.3389/fonc.2011.00030

27. Walczak M, Martens S. Dissecting the role of the Atg12-Atg5-Atg16 complex during autophagosome formation. Autophagy. 2013;9 (3):424-425. doi:10.4161/auto.22931

28. Eisenberglerner A, Bialik S, H U S, et al. Life and death partners: apoptosis, autophagy and the cross-talk between them. Cell Death Differ. 2009;16(7):966-975. doi:10.1038/cdd.2009.33

29. Mariño G, Niso-Santano M, Baehrecke EH, et al. Self-consumption: the interplay of autophagy and apoptosis. Nat Rev Mol Cell Biol. 2014;15:81-94. doi:10.1038/nrm3735

30. Fu LL, Cheng Y, Liu B. Beclin-1: autophagic regulator and therapeutic target in cancer. Int J Biochem Cell Biol. 2013;45:921-924. doi:10.1016/j.biocel.2013.02.007

31. Lindqvist LM, Heinlein M, Huang DC, et al. Prosurvival Bcl-2 family members affect autophagy only indirectly,by inhibiting Bax and Bak. Proc Natl Acad Sci USA. 2014;111:8512-8517. doi:10.1073/pnas.1406425111

32. Yousefi S, Perozzo R, Schmid I, et al. Calpain-mediated cleavage of Atg5 switches autophagy to apoptosis. Nat Cell Biol. 2006;8:11241132. doi:10.1038/ncb1482

33. Rubinstein AD, Eisenstein M, Ber Y, et al. The autophagy protein Atg12 associates with antiapoptotic Bcl-2 family members to promote mitochondrial apoptosis. Mol Cell. 2011;44:698-709. doi:10.1016/j.molcel.2011.10.014

34. Li HY, Zhang J, Sun L, et al. Celastrol induces apoptosis and autophagy via the ROS/JNK signaling pathway in human osteosarcoma cells: an in vitro and in vivo study. Cell Death Dis. 2015;6(1): e1604. doi:10.1038/cddis.2014.543

35. Wang H, Zhang T, Sun W, et al. Erianin induces G2/M-phase arrest, apoptosis, and autophagy via the ROS/JNK signaling pathway in human osteosarcoma cells in vitro and in vivo. Cell Death Dis. 2016;7(6):e2247. doi:10.1038/cddis.2016.138

36. Liu J, Chang F, Li F, et al. Palmitate promotes autophagy and apoptosis through ROS-dependent JNK and p38 MAPK. Biochem Biophys Res Commun. 2015;463(3):262-267. doi:10.1016/j. bbrc.2015.05.042

37. Zorov DB, Juhaszova M, Sollott SJ. Mitochondrial reactive oxygen species (ROS) and ROS-induced ROS release. Physiol Rev. 2014;94 (3):909-950. doi:10.1152/physrev.00026.2013

38. Wang C, Youle RJ. The role of mitochondria in apoptosis*. Annu Rev Genet. 2009;43:95-118. doi:10.1146/annurev-genet-102108-134850

39. Redza-Dutordoir M, Averill-Bates DA. Activation of apoptosis signalling pathways by reactive oxygen species. Biochim Biophys Acta. 2016;1863(12):2977-2992. doi:10.1016/j.bbamcr.2016.09.012 
40. Gong K, Chen C, Zhan Y, Chen Y, Huang Z, Li W. Autophagyrelated gene 7 (ATG7) and reactive oxygen species/extracellular signal-regulated kinase regulate tetrandrine-induced autophagy in human hepatocellular carcinoma. $J$ Biol Chem. 2012;287 (42):35576-35588. doi:10.1074/jbc.M112.370585

41. Sato A, Okada M, Shibuya K, et al. Pivotal role for ROS activation of p38 MAPK in the control of differentiation and tumor-initiating capacity of glioma-initiating cells. Stem Cell Res. 2014;12(1):119131. doi:10.1016/j.scr.2013.09.012
42. Xu J, Qin X, Cai X, et al. Mitochondrial JNK activation triggers autophagy and apoptosis and aggravates myocardial injury following ischemia/reperfusion. Biochim Biophys Acta. 2015;1852 (2):262-270. doi:10.1016/j.bbadis.2014.05.012

43. Shieh JM, Huang TF, Hung CF, Chou KH, Tsai YJ, Wu WB. Activation of c-Jun N-terminal kinase is essential for mitochondrial membrane potential change and apoptosis induced by doxycycline in melanoma cells. Br J Pharmacol. 2010;160(5):1171-1184. doi:10.1111/j.14765381.2010.00746.x 


\section{Supplementary materials}

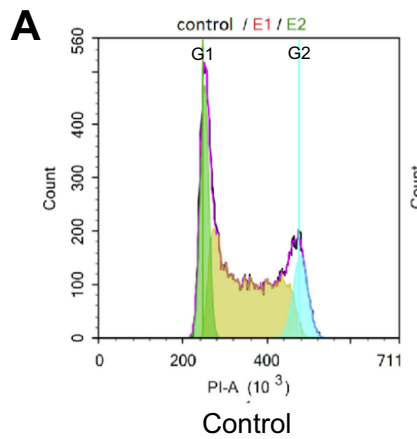

B U202 cells
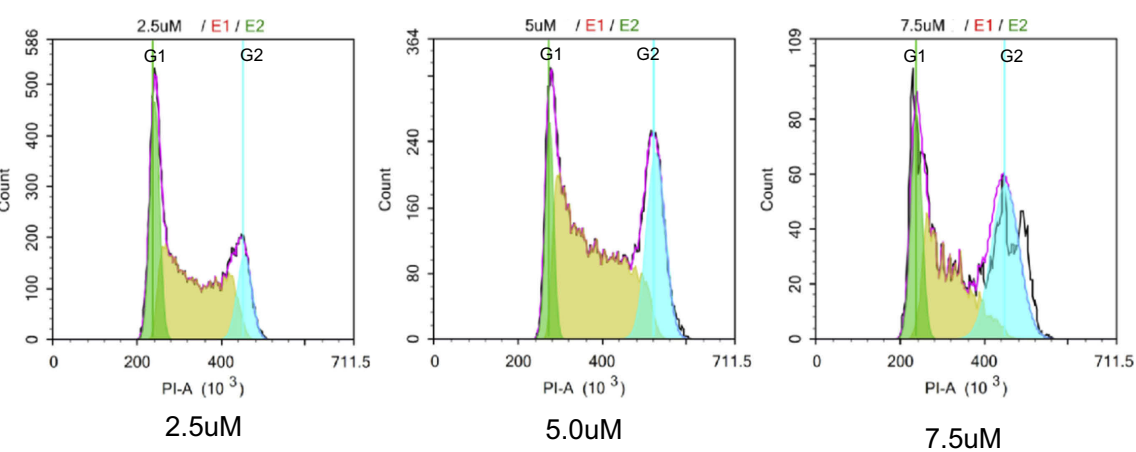

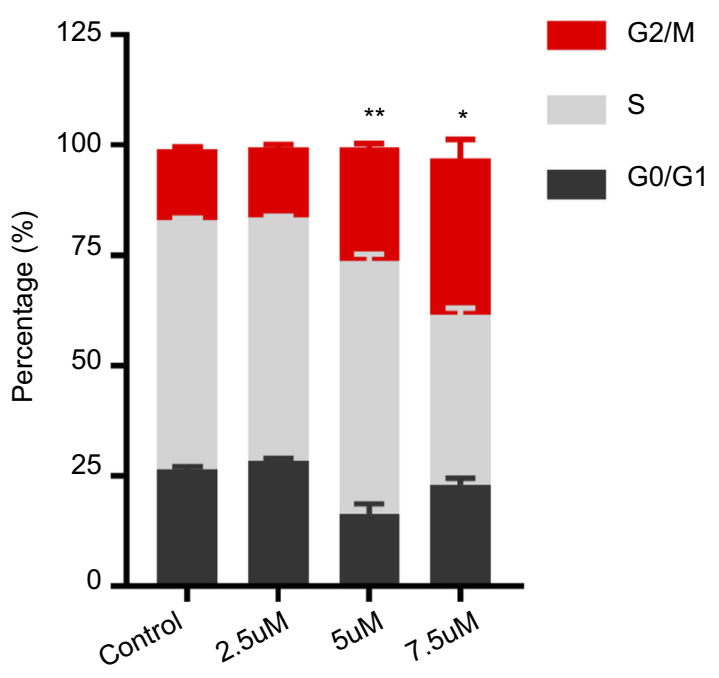

Polyphyllin VI concentration

Figure SI Polyphyllin VI induces G2/M cell cycle arrest. (A) The alterations in cell cycle distribution of U2OS cells were determined by flow cytometry after treatment with control and Polyphyllin VI $(2.5,5$, and $7.5 \mu \mathrm{M})$ for $24 \mathrm{~h}$. (B) Ratios of cells in different cell cycle phases in U2OS cells. The percentage of cells in each phase is shown as mean $\pm \mathrm{SD}$ from three independent experiments. $* \mathrm{P}<0.05$ and $* * \mathrm{P}<0.0 \mathrm{I}$, significantly different compared with control.
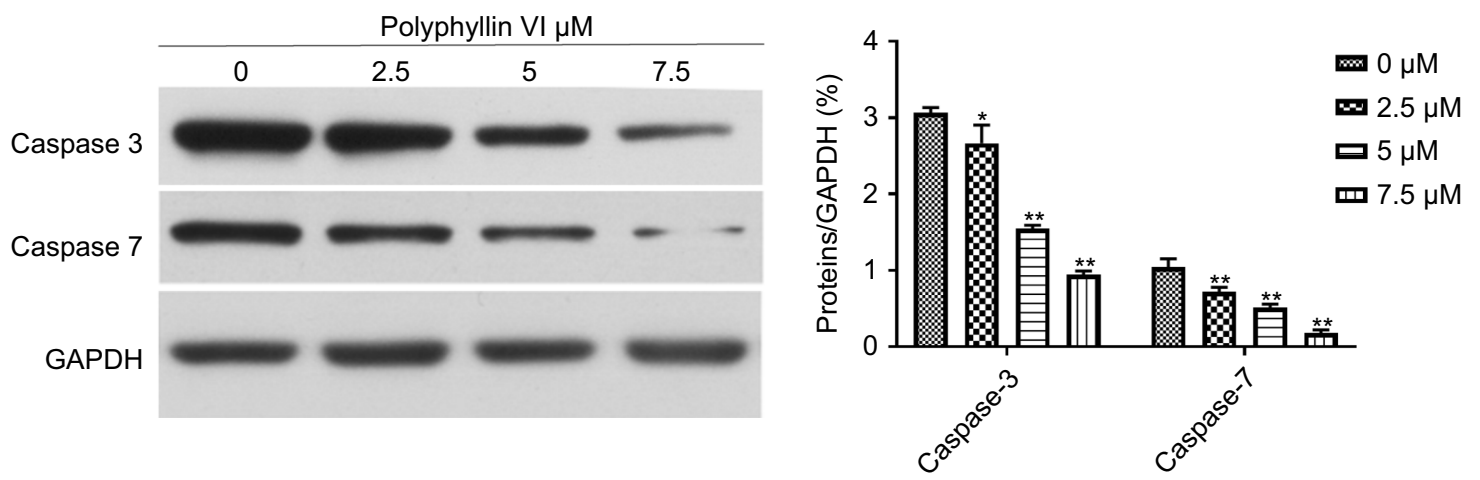

Figure S2 U2OS cells were incubated with control and Polyphyllin VI $(2.5,5$, and $7.5 \mu \mathrm{M})$ for $24 \mathrm{~h}$. Cell lysates were prepared and analyzed by Western blotting for Caspase- 3 and Caspase-7. Results are presented as the mean \pm SD from three independent experiments. $* P<0.05$ and $* * P<0.0$, significantly different compared with control. 


\section{Publish your work in this journal}

Drug Design, Development and Therapy is an international, peerreviewed open-access journal that spans the spectrum of drug design and development through to clinical applications. Clinical outcomes, patient safety, and programs for the development and effective, safe, and sustained use of medicines are a feature of the journal, which has also been accepted for indexing on PubMed Central. The manuscript management system is completely online and includes a very quick and fair peer-review system, which is all easy to use. Visit http://www. dovepress.com/testimonials.php to read real quotes from published authors. 